\title{
Analysis of Inclined Strip Anchors in Sand Based on the Block Set Mechanism
}

\author{
S. B. Yu ${ }^{1, a}$, J. P. Hambleton ${ }^{1, b}$, and S. W. Sloan ${ }^{1, c}$ \\ ${ }^{1} \mathrm{ARC}$ Centre of Excellence for Geotechnical Science and Engineering, The University of Newcastle, \\ Australia. \\ aShengbing.Yu@Newcastle.edu.au, bJames.Hambleton@Newcastle.edu.au, \\ cScott.Sloan@Newcastle.edu.au
}

Keywords: anchors; sand; failure surface; block set mechanism; limit analysis

\begin{abstract}
Anchors are widely used in foundation systems for structures requiring uplift resistance. As demonstrated by numerous theoretical and experimental studies on the subject, uncertainty remains as to both the theoretical uplift capacity of anchors in idealised soils and the suitability of the various modelling assumptions in capturing the responses observed during tests. This study, which deals exclusively with the theoretical uplift capacity, presents newly obtained predictions of uplift capacities and the corresponding collapse mechanisms for inclined strip anchors in sand. The analysis is based on the upper bound (kinematic) method of limit analysis and the so-called block set mechanism, in which a collapse mechanism consisting of sliding rigid blocks is optimised with respect to interior angles and edges of the blocks. It is demonstrated that the method provides lower (better) estimates of uplift capacity in some cases compared to previous upper bound calculations. Also, variations in the predicted collapse mechanism with changes in embedment and inclination are assessed in detail.
\end{abstract}

\section{Introduction}

Plate anchors are commonly used for transmission towers, earth-retaining walls, mooring systems for offshore facilities, and other applications where the foundation system is subjected to uplift. Anchors can be installed in either sand or clay, and they appear in various configurations. In this study, attention is restricted to the case of sand and thin rectangular (strip) anchors for which the length is significantly greater than the width, such that plane strain can be assumed. While the assumption of plane strain may be unrealistic for many applications, this case is essential as a reference, in much the same way as the strip footing is a reference in the assessment of bearing capacity of shallow foundations. Furthermore, the aim of this study is to assess the theoretical ultimate uplift capacity of strip anchors in sand, and, in accordance with classical plasticity theory, it is assumed that the material is perfectly plastic and can be characterised by the Mohr-Coulomb yield condition with associated plastic flow. The material is taken to be purely frictional, so that the only strength parameter is the angle of internal friction $\varphi$.

Numerous studies on the uplift capacity of strip anchors in sand can be found in the literature. Many of these involve the use of the limit equilibrium method [1-4], or are based on formulae derived empirically from laboratory model tests [5-9]. Although the limit equilibrium method provides a simple and useful way of analyzing the stability of geotechnical structures, an arbitrary failure surface must be assumed a priori to calculate the collapse load. Alternative solutions have been obtained using the displacement-based finite element method [6], cavity expansion theory [10], and limit analysis [8, 11-15].

Among the previous investigations pertaining to strip anchors in sand, relatively few have examined the effects of anchor inclination. This important case occurs in applications where loads are applied at some inclination from the vertical (e.g., transmission towers), and the anchor itself is installed at an angle in order to maximise uplift capacity. The present study builds on previous works based on limit analysis of inclined anchors in sand $[8,12,14]$ by proposing a new type of collapse mechanism, which in some cases yields improved predictions of uplift capacity. The analysis also 
provides insight into how the collapse mechanism changes with the anchor embedment ratio and inclination of the anchor.

\section{Problem Definition}

A schematic of the problem considered in this study is shown in Fig. 1. The relevant variables are the ultimate force at failure $Q_{u}$, the width of the anchor $B$, the angle of inclination $\alpha$, and the depth of embedment $H$, which is defined as the vertical distance from the midpoint of the strip anchor to the ground surface. As depicted, it is assumed that the direction of pullout is perpendicular to the anchor face, and the plate anchor is taken to be rigid and have zero thickness.

For inclined anchors, active failure may develop behind the anchor, which tends to reduce the capacity. However, as shown by Merifield [14], active failure behind the anchor is potentially significant only when both the embedment ratio and the friction angle are low (roughly when $H / B \leq 2$ and $\varphi \leq 20^{\circ}$ ). For most cases, the effect of active failure appears to be small, or the collapse mechanism consists only of passive failure of soil. In this study, only the passive failure of soil ahead of the anchor is considered.

The ultimate uplift capacity of anchors in sand can be expressed in the following form

$$
q_{u}=\frac{Q_{u}}{A}=\gamma H N_{\gamma}
$$

where $A$ is the area of anchor, $\gamma$ is the soil unit weight, and $N_{\gamma}$ is the uplift capacity factor, which depends on the embedment ratio $H / B$, inclination angle $\alpha$, and friction angle $\varphi$.

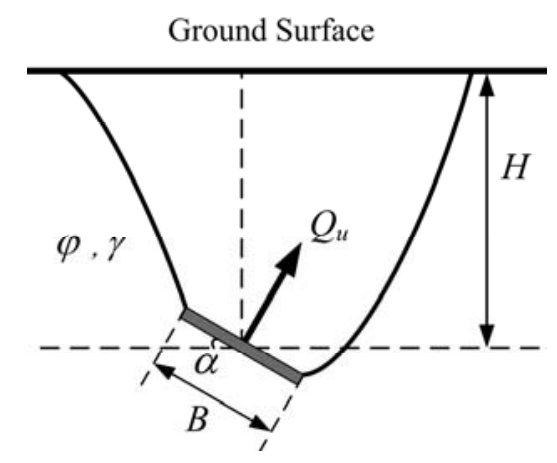

Fig. 1. Problem notation for inclined anchor in sand.

\section{Upper Bound Limit Analysis Based on Block Set Mechanism}

The collapse mechanism assumed in this study consists of sliding rigid blocks separated by infinitesimally thin regions undergoing shear (cf. [16]). As shown in Fig. 2, a kinematically admissible velocity field is constructed using two groups of rigid blocks referred to as "combination sets". In the figure, half of the total number of blocks are shown for clarity, and the vertices of triangular rigid blocks are denoted by circles (1st combination set) or triangles (2nd combination set). By equating the rate of work done by external forces to the rate of plastic dissipation, an upper bound on the true uplift capacity can be ascertained. Since the material is purely frictional, dissipation occurs only due to frictional contact at the anchor-soil interface, which is taken to be fully rough (interface friction angle $\varphi$ ).

Since the predicted uplift capacity is an upper bound, the objective is to find the geometry of the mechanism that minimizes the uplift capacity. This was achieved by treating that interior angles and edge lengths of the blocks as unknowns, and subsequently optimizing these variables using a hybrid genetic algorithm (GA) combined with a pattern search method (or direct search method). The number of blocks is determined adaptively, such that the final mechanism can include hundreds of blocks. Full details of the method, referred to as the "block set mechanism", are given by Yu [17]. 
Ground Surface

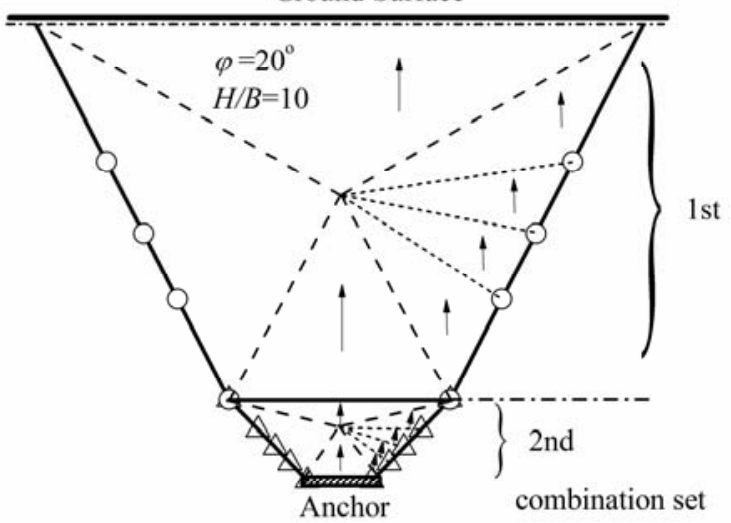

Ground Surface

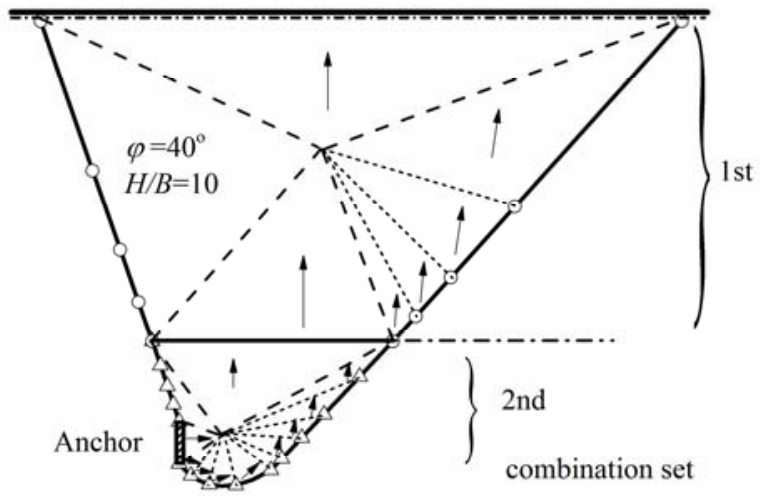

Fig. 2. Collapse mechanism for (a) horizontal anchor and (b) vertical anchor (velocity vectors are illustrative only).

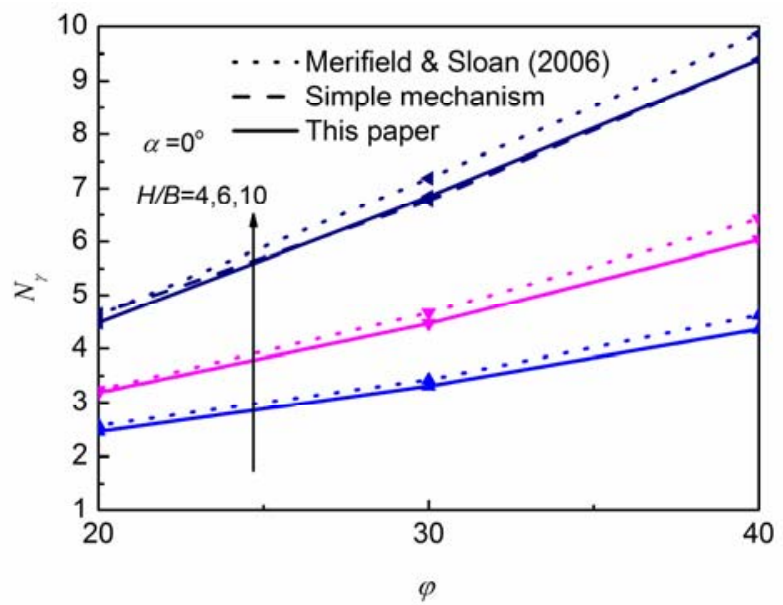

(a) $\alpha=0^{\circ}$

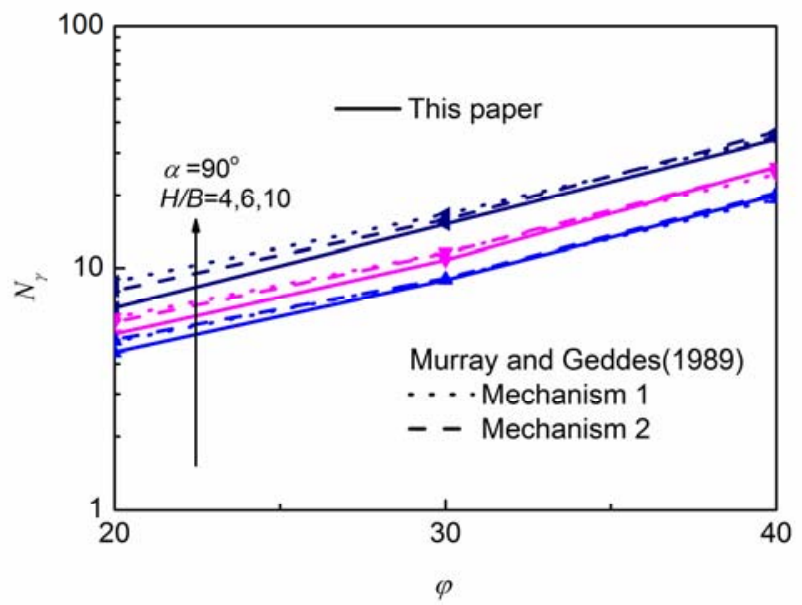

(b) $\alpha=90^{\circ}$

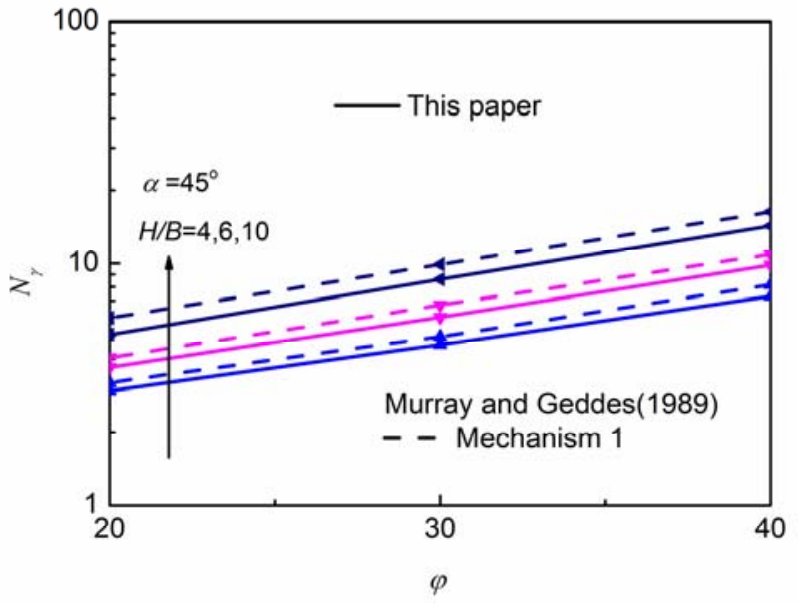

(c) $\alpha=45^{\circ}$

Fig. 3. Comparison of calculated uplift capacities with previous solutions. Note that vertical scale is linear in (a) and logarithmic in (b) and (c). 


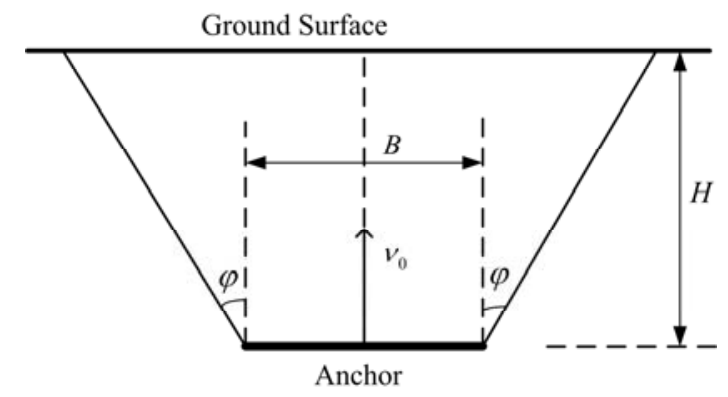

Fig. 4. Simple mechanism for horizontal anchor in sand.

\section{Uplift Capacities}

Comparison of calculated uplift capacities with existing upper bound solutions is shown in Fig. 3. For a horizontal anchor $(\alpha=0)$, it can be seen that the calculated values are very close to those obtained using the "simple mechanism" shown in Fig. 4, for which $N_{\gamma}=1+(H / B) \tan \varphi$. The upper bounds are also smaller than those obtained by Merifield and Sloan [12] using finite element limit analysis. For the inclined anchors $\left(\alpha=45^{\circ}\right.$ and $\left.90^{\circ}\right)$, comparison is made with the upper bounds assessed using the two mechanisms proposed by Murray \& Geddes [8], where "mechanism 1" consists of two sliding rigid blocks and "mechanism 2" includes two blocks separated by a zone of continuous shear. Only the results for "mechanism 1" are shown for $\alpha=90^{\circ}$ (Fig. 3(c)), since they provide smaller upper bounds than "mechanism 2". It can be seen that in most case the values obtained in the present current study are lower than those obtained with the mechanisms of Murray and Geddes (1989).

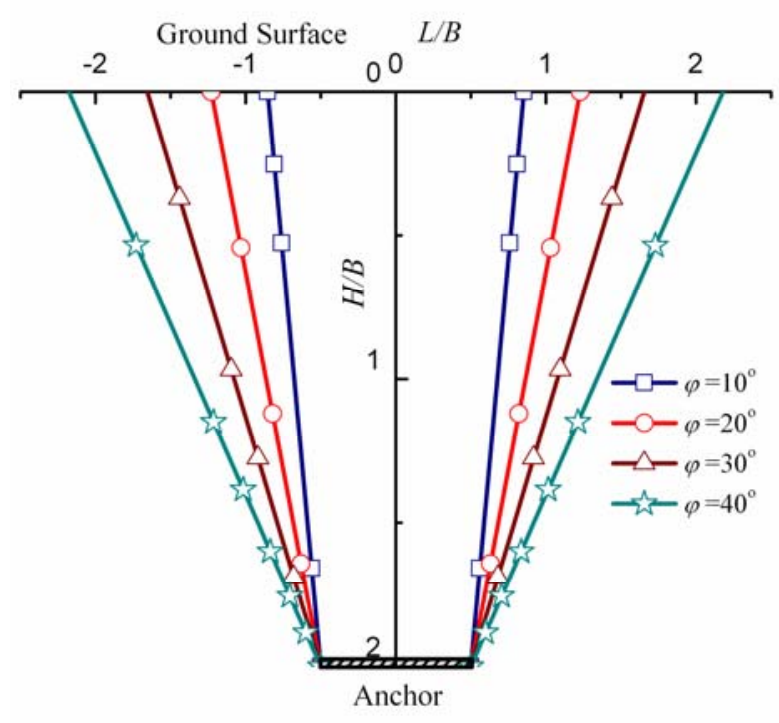

(a) $H / B=2$

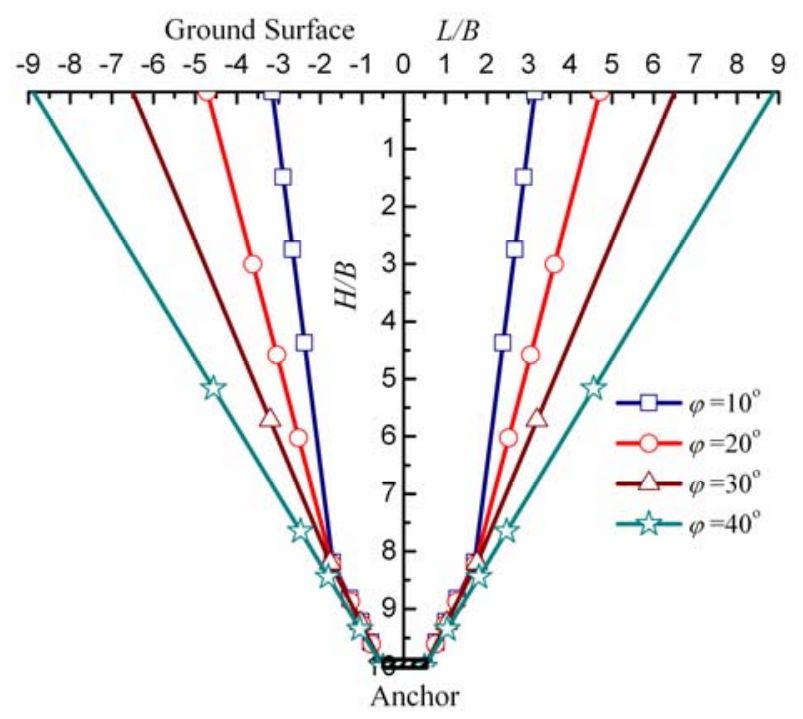

(b) $H / B=10$

Fig. 5. Failure surfaces for horizontal anchors $(\alpha=0)$.

\section{Collapse Mechanisms}

The failure surfaces (i.e., bounding slip surfaces for the optimized mechanism) for horizontal anchors with different embedment ratios $(H / B)$ and friction angles are shown in Fig. 5. Here $L$ is the distance in the horizontal direction, and unequal vertical and horizontal scales are used. Generally, the failure surfaces obtained from the block set mechanism and the "simple mechanism" shown in Fig. 4 are very similar. Somewhat surprisingly, however, it can be seen that differing failure surfaces 
are obtained for large embedment ratios $(H / B>7)$ and lower values of friction angle $\varphi$. This point is confirmed upon comparison of the uplift capacities shown in Fig. 3(a), which reveals that results obtained using the block set mechanism are lower than that of simple mechanism for $H / B=10$ and $\varphi$ $=20^{\circ}$.

Failure surfaces for inclined anchors are shown in Fig. 6 for two different cases, where the figure emphasizes potential variations in the failure surface with depth. A basic conclusion is that the shape of the failure surface varies little with increasing embedment ratio, which may assist in the development of analytical solutions.

Figure 7 emphasizes variations in the failure surface as a function of inclination angle. It can be seen that the lateral extent of failure surface becomes larger with increasing inclination angle. As the inclination changes from horizontal to vertical, the failure surface changes dramatically. The left side of failure surface basically remains straight, while the right side becomes increasingly curved as the inclination angle increases.

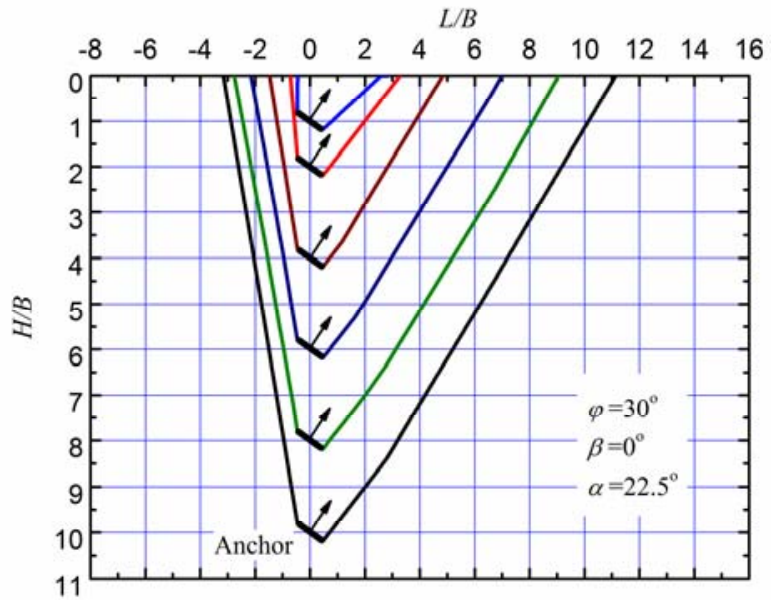

(a) $\alpha=22.5^{\circ}$

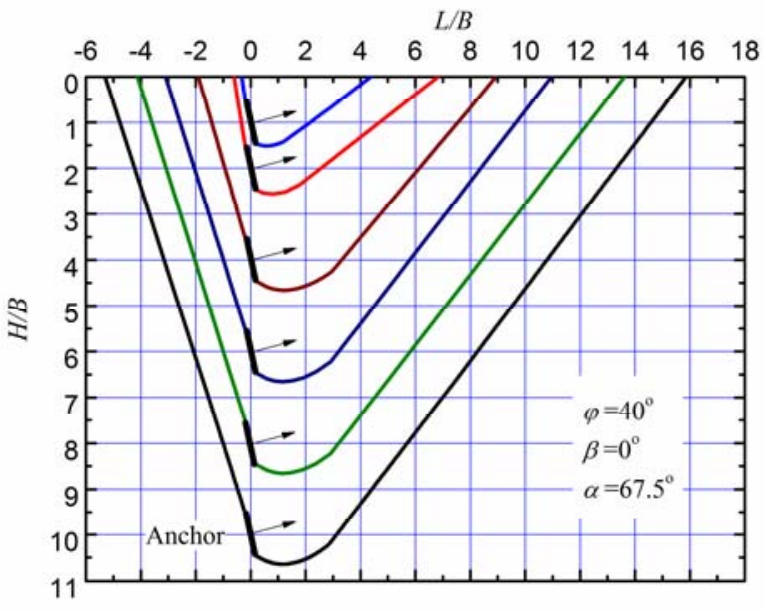

(b) $\alpha=67.5^{\circ}$

Fig. 6. Failure surfaces for inclined anchors at different embedment ratios.

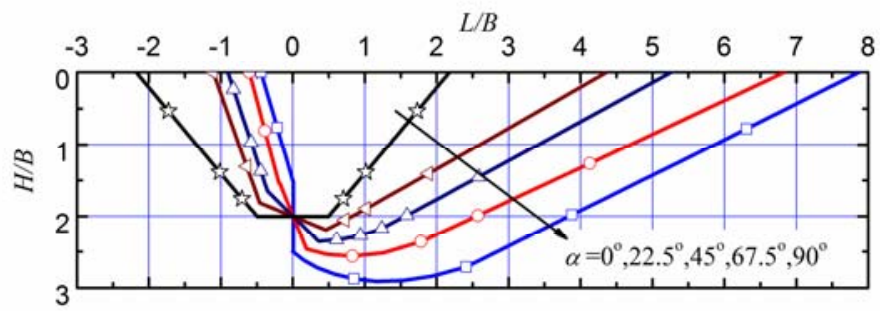

(a) $H / B=2$

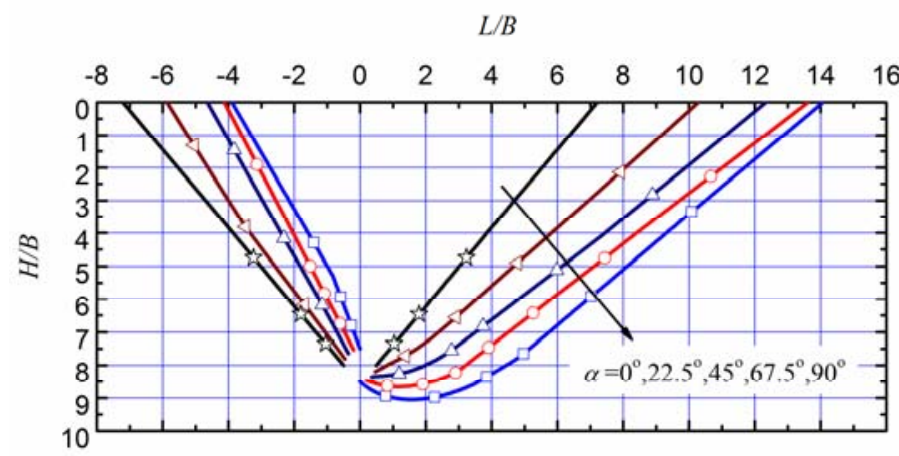

(b) $H / B=8$

Fig. 7. Failure surfaces for inclined anchors at different inclination angles $\left(\varphi=40^{\circ}\right)$. 


\section{Concluding Remarks}

The paper presents new upper bounds on the uplift capacity of inclined anchors in purely frictional material (sand). These are obtained using the block set mechamism proposed by $\mathrm{Yu}$ [17]. Consideration is mainly given to the effects of anchor embedment ratio and inclination angle, parameters which have a great impact on the uplift capacity and the shape of the collapse mechanism. The uplift capacities obtained using the block set mechanism are generally less than those obtained previously, and insights drawn by comparing the failure surfaces for the various cases may assist in future development of analytical solutions. The results can provide a useful check on collapse loads obtained from elastoplastic finite element analysis, and, from a practical viewpoint, the predicted failure surfaces can provide the engineer with a physical feeling about how the soil mass may fail.

\section{References}

[1] G.G. Meyerhof, J.I. Adams, The ultimate uplift capacity of foundations, J. Canadian Geotechnical Journal, 1968, 5(4): 225-244.

[2] D.J. White, C.Y. Cheuk, M.D. Bolton, The uplift resistance of pipes and plate anchors buried in sand, J. Geotechnique, 2008, 58(10): 771-779.

[3] D. Choudhury, K.S. Subba Rao, Seismic uplift capacity of inclined strip anchors, J. Canadian Geotechnical Journal, 2005, 42(1): 263-271.

[4] D. Choudhury, K.S. Subba Rao, Generalized solution for seismic uplift capacity of strip anchors using pseudo-static approach, J. Journal of Earthquake and Tsunami, 2007, 1(4): 311-328.

[5] B.M. Das, G.R. Seeley, Breakout Resistance of Shallow Horizontal Anchors, J. Journal of the Geotechnical Engineering Division, 1975, 101(9): 999-1003.

[6] R.K. Rowe, E.H. Davis, The behaviour of anchor plates in sand, J. Geotechnique, 1982, 32(1): $25-41$.

[7] E.A. Dickin, M. Laman, Uplift response of strip anchors in cohesionless soil, J. Advances in Engineering Software, 2007, 38(8-9): 618-625.

[8] E.J. Murray, J.D. Geddes, Resistance of passive inclined anchors in cohesionless medium, J. Geotechnique, 1989, 39(3): 417-431.

[9] S. Frydman, I. Shaham, Pullout capacity of slab anchors in sand, J. Canadian Geotechnical Journal, 1989, 26(2): 385-400.

[10]H.S. Yu, Cavity expansion methods in geomechanics, Kluwer Academic Publishers, 2000.

[11]R.S. Merifield, A.V. Lyamin, S.W. Sloan, Three-dimensional lower-bound solutions for the stability of plate anchors in sand, J. Geotechnique, 2006, 56(2): 123-132.

[12]R.S. Merifield, S.W. Sloan, The ultimate pullout capacity of anchors in frictional soils, J. Canadian Geotechnical Journal, 2006, 43(8): 852-868.

[13] J. Kumar, Upper bound solution for pullout capacity of anchors on sandy slopes, J. International Journal for Numerical and Analytical Methods in Geomechanics, 1997, 21(7): 477-484.

[14]R.S. Merifield, Numerical modelling of soil anchors, University of Newcastle, Australia 2002.

[15]P. Regenass, A.H. Soubra, Passive resistance of strip anchors, Anchors in theory and practice.

Balkema, Rotterdam. 1995: 117-123.

[16] W. F, Chen. Limit Analysis and Soil Plasticity, Elsevier, Amsterdam, 1975.

[17] S. B. Yu, Block set mechanism for upper bound analysis and its applications, Tongji University, 2011. 